\title{
Antenna Design for Underwater Radio Communications
}

\author{
S. I. Inácio, M. R. Pereira, H. M. Santos, L. M. Pessoa, F. B. Teixeira, M. J. Lopes, O. Aboderin, H. M. Salgado \\ INESC TEC and Faculdade de Engenharia, Universidade do Porto \\ Rua Dr. Roberto Frias, s/n \\ 4200-465 Porto, Portugal \\ sofia.inacio@fe.up.pt, \{mario.r.pereira, hugo.m.santos, luis.m.pessoa, fbt, mjlopes, oluyomi.aboderin, hsalgado\}@inesctec.pt
}

\begin{abstract}
In this paper we assess the influence of the conductivity of the medium on the radiation characteristics and input impedance of a loop antenna designed for underwater communications. The initial study is based on simulation of the antenna characteristics using FEKO electromagnetic (EM) software. Additionally, an equivalent circuit model of the antenna is also obtained and simulation of the input impedance on ADS software shows good agreement with the EM simulator. It is found that the radiation pattern, of the loop antenna changes significantly with the conductivity of the medium, from freshwater to seawater. The loop antennas were built with baluns and tested in freshwater, where the insertion loss between two identical antennas and the radiation pattern of one of them were also measured. The results show good agreement with the simulations.
\end{abstract}

Index Terms-underwater communications, RF communications, antennas, radiation pattern, balun.

\section{INTRODUCTION}

Acoustic technologies have been the standard for underwater wireless communications. Although they are suitable for long range communications (of the order of tens of kilometres), they typically imply high latency, low data-rates and poor noise immunity [1]. These characteristics make them unfit for real-time and broadband underwater wireless sensor networks, that are becoming an attractive approach for a broad range of industries, including defence, offshore oil and gas, as well as for environmental monitoring.

Underwater electromagnetic (EM) communications were intensively investigated in the $60 \mathrm{~s}$, with only a few practical applications, since the huge attenuation of water limits the system range to a few meters.

Underwater optical wireless communications are considered an interesting alternative to acoustic systems, since they allow high data-rates and low latency. However those systems are affected by suspended particles and marine fouling [2], whereas Radio Frequency (RF) waves are immune to dirty water conditions. Additionally, the range of underwater RF technology can be increased when operating near the surface. In fact, results have shown that effective communication between a submerged node and land is possible without the need for surface repeater buoys, since the air path carries most of the signal [3]. Additionally, the Wireless For Subsea company has been releasing underwater radio-frequency modems since 2006, which demonstrates the rise in interest in this technology [4].

The propagation properties of EM waves underwater make them especially well-suited for short-range broadband communications. One important application is the real-time guidance of an autonomous underwater vehicle (AUV) when approaching an underwater docking station and subsequent high-speed data transfer between the AUV and the dock. The ENDURE project, lead by INESC TEC and funded by EEA Grants, targets the demonstration and feasibility of such system, in order to enable the unattended operation of AUVs in remote oceanic locations for extended periods of time. Another important application is the implementation of IEEE 802.11 networks in freshwater and seawater, that was evaluated in [5] and in [6] at VHF, UHF and SHF bands with the help of softwaredefined radios. Previous research on the physical layer of RF underwater communications has been focused mainly in channel characterization. In fact, research on antenna design for underwater operation has been scarce, and an analysis of the influence of the different structural antenna parameters for both fresh and seawater has never been presented.

The rest of this paper is organized as follows. Sections 2 and 3 present the relevant water properties, and the theoretical model considered, respectively. In section 4 an analysis of the loop antenna is presented, taking in to account the conductivity of the medium, based on simulations in FEKO and ADS software. Section 5 presents the experimental procedure and the results of the tests made in a freshwater pool, while the main conclusions are addressed in section 6 .

\section{WATER PROPERTIES}

The best media for electromagnetic waves propagation are insulators, where the conductivity is zero $(\sigma=0 \mathrm{~S} / \mathrm{m})$. In those media, electromagnetic waves are not attenuated and therefore they are known as lossless media [7]. If the conductivity of the medium increases, the attenuation of radio waves also increases [8].

Freshwater conductivity can range from 0.005 to $0.05 \mathrm{~S} / \mathrm{m}$ [9], the actual value increasing with salinity and temperature. Thus, seawater has a higher conductivity, with an average of $4 \mathrm{~S} / \mathrm{m}$. 
In a medium with a conductivity $\sigma$ and at the angular frequency $\omega$, the permittivity becomes complex, with a value of [7]:

$$
\epsilon=\epsilon_{r} \epsilon_{0}-j \frac{\sigma}{\omega}=\epsilon^{\prime}-j \epsilon^{\prime \prime} \mathrm{F} / \mathrm{m}
$$

where $\epsilon_{0}$ is the vacuum permittivity.

The relative permittivity $\left(\epsilon_{r}\right)$ of water depends upon several factors like water temperature, salinity and propagation frequency and it can be described by the Debye model [10], [11] or by the Cole-Cole equation [12], [13]. In this work we considered a relative permittivity value of 81 for both fresh and seawater, since according to the models presented above that is the value of the water permittivity in the frequency range of interest for this work (1 to $100 \mathrm{MHz}$ ).

Since water is not a magnetic medium the value of its relative permeability is $\mu_{r}=1$. So the permeability $(\mu)$ of water is the same as that of free space.

\section{Propagation Models for Underwater Media}

The propagation of electromagnetic waves, in any medium, is characterized by its propagation constant, $\gamma$, which is given by [7]:

$$
\gamma=\sqrt{j \omega \mu\left(\sigma+j \omega \epsilon^{\prime}\right)}=\alpha+j \beta
$$

where $\alpha(\mathrm{Np} / \mathrm{m})$ and $\beta(\mathrm{rad} / \mathrm{m})$ are the attenuation and phase constants, respectively, and $\omega$ is the angular frequency.

Media where $\frac{\sigma}{\omega \epsilon^{\prime}} \ll 1$ are considered dielectric media, or insulators. On the other hand, media where $\frac{\sigma}{\omega \epsilon^{\prime}} \gg 1$ are considered conductors [14]. In Fig. 1 the behaviour of $\frac{\sigma}{\omega \epsilon^{\prime}}$ is shown as a function of frequency for the two media considered in this work. It can be seen that freshwater becomes a conductor for frequencies below $10 \mathrm{MHz}$ and in the case of seawater this transition occurs at $900 \mathrm{MHz}$.

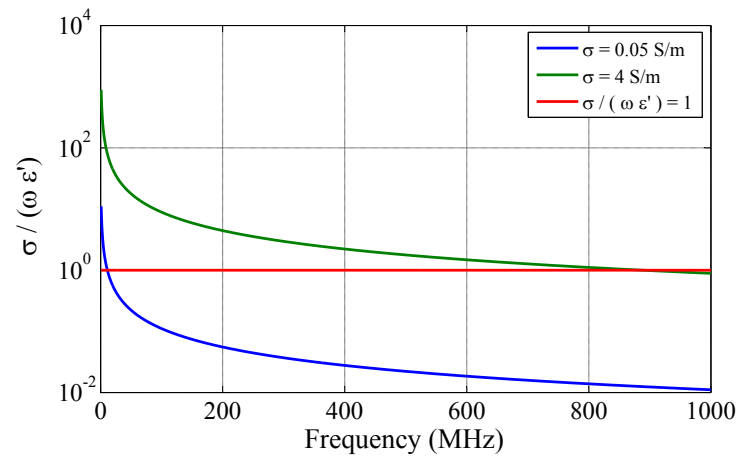

Fig. 1. Behavior of $\frac{\sigma}{\omega \epsilon^{\prime}}$ as a function of frequency for the two media considered in this work $(\sigma=0.05 \mathrm{~S} / \mathrm{m}$ e $\sigma=4 \mathrm{~S} / \mathrm{m})$.

Fig. 2 shows the relation between the attenuation of an electromagnetic wave and its frequency, in freshwater and seawater. It can be observed that attenuation increases with the frequency and with the conductivity. So, we can conclude that it is necessary to use frequencies under $100 \mathrm{MHz}$ to get a reasonable communication range, especially in seawater where the attenuation is more severe.

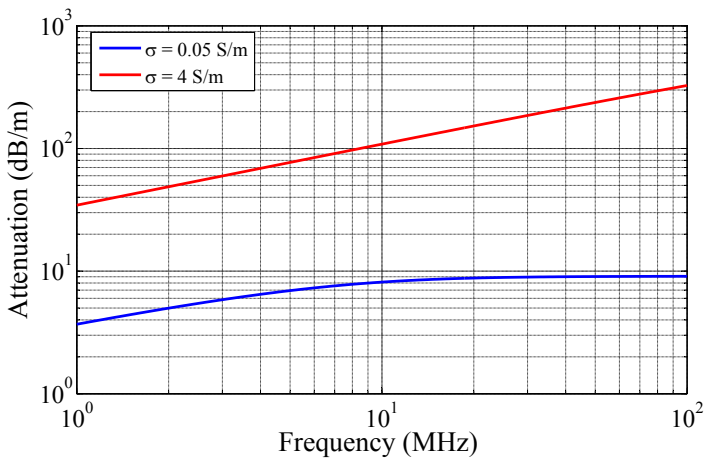

Fig. 2. Attenuation of an electromagnetic wave propagating in two different media $(\sigma=0.05 \mathrm{~S} / \mathrm{m}$ e $\sigma=4 \mathrm{~S} / \mathrm{m})$

\section{Antennas in Underwater Media}

In this section we assess through simulation the performance of a loop antennas both in fresh and seawater, in terms of resonance frequency, input impedance and radiation pattern. This type of antenna was selected and implemented since it is simple to produce at a low-cost and was already validated in others projects carried out at INESC TEC: in [5] and in [6] a loop antenna $5 \mathrm{~cm}$ radius was designed for underwater environments and its operation was verified successfully. So, in this work, we improved its design to better adapt it to the underwater environment.

The loop antenna is depicted in Fig. 3 and consisted of a simple $3 \mathrm{~mm}$ thick cooper wire loop, with a radius of $16 \mathrm{~cm}$, covered with an insulator with a thickness of $50 \mu \mathrm{m}$ and a relative permittivity of 3 .

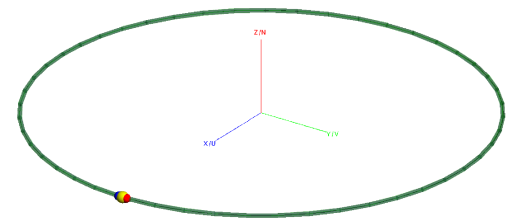

Fig. 3. Underwater loop antenna model.

\section{A. Resonance Frequency and Radiation Pattern}

We performed an extensive analysis of this loop antenna using the FEKO 3D electromagnetic simulator. Fig. 4 shows the dependency of two major antenna parameters as a function of water conductivity, namely the resonance frequency (Fig. 4(a)) and the real part of the impedance at that frequency (Fig. 4(b)). From Fig. 4 it is clearly seen that both the resonance frequency and the input impedance of the loop antenna change dramatically with the conductivity of water. From these results we readily conclude that the same physical antenna will not be suitable for both fresh and seawater environments, as the resonance frequency is relatively different. Moreover, from Fig. 4(b) we can also conclude that depending on the 


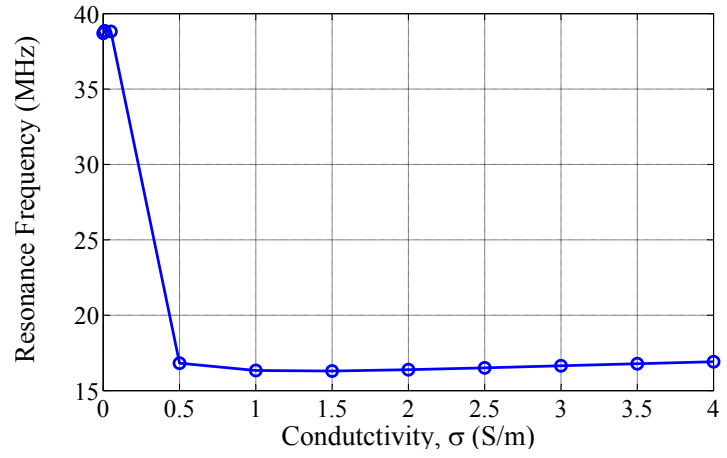

(a)

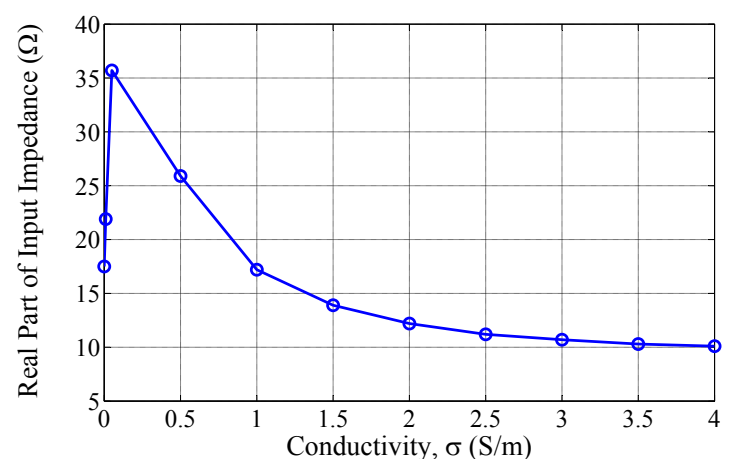

(b)

Fig. 4. Dependency of: (a) resonance frequency and (b) real part of input impedance at resonance, on the water conductivity.

conductivity of water, different matching networks must be designed, for an efficiently use of the antenna.

Fig. 5(a) and Fig. 5(b) show the 3D radiation patterns for the loop antenna, with the antenna placed in the same orientation, for freshwater and seawater, respectively. Again we see the influence of water conductivity on the performance of the antenna. In fresh water the radiation pattern maximums are oriented in the $\mathrm{z}+$ and $\mathrm{z}$ - directions, whereas in seawater, they are shifted by $90^{\circ}$.

\section{B. Equivalent Circuit}

The equivalent circuit of a loop antenna in a lossy medium can be modelled using the eight lumped elements shown in Fig. 6. The equivalent circuit is based on the five lumped elements circuit of a magnetic antenna in free space showed in [15] and on the method presented in [16] for the equivalent circuit of a dipole in a lossy medium, that states that in a lossy medium all capacitors become conductive and the resistor becomes inductive.

Knowing the first and second resonant frequencies of the antenna in free space, we started by obtaining the values of the five lumped elements of the equivalent circuit. Then we used the expressions presented in [16], considering the properties of the lossy medium where the antenna was placed, to obtain the values of the three remaining elements.

Fig. 7 compares the simulation results of the equivalent circuit of Fig. 6 for the first resonant frequency of the

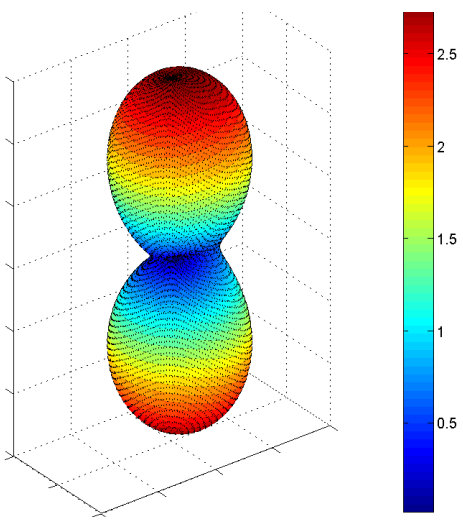

(a)

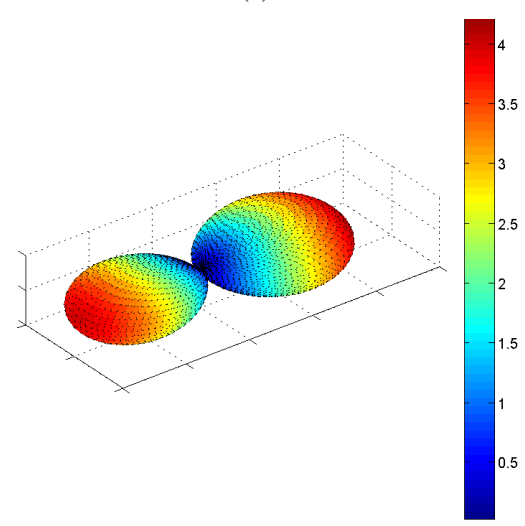

(b)

Fig. 5. 3D radiation patterns of the loop antenna: (a) in a medium with $\sigma=0.05 \mathrm{~S} / \mathrm{m}$ and (b) in a medium with $\sigma=4 \mathrm{~S} / \mathrm{m}$.

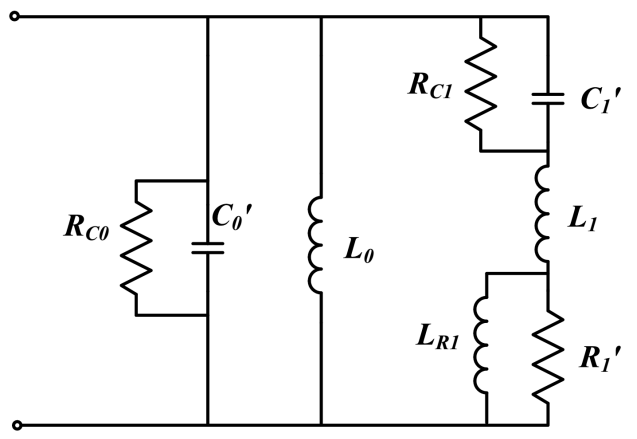

Fig. 6. Equivalent circuit of the loop antenna in a lossy medium.

uninsulated loop antenna in freshwater and the simulation results obtained in FEKO for the same antenna. The values of the lumped elements of the circuit in Fig. 6 are shown in Table I. The values of $R_{1}^{\prime}$ and $L_{R 1}$ are frequency dependent, and are given by:

$$
\begin{gathered}
R_{1}^{\prime}=\frac{3.75}{\cosh \left(0.5 \sinh ^{-1}\left(\frac{1.11 \times 10^{7}}{f}\right)\right)} \Omega \\
L_{R 1}=\frac{3.75}{2 \pi f \cdot \cosh \left(0.5 \sinh ^{-1}\left(\frac{1.11 \times 10^{7}}{f}\right)\right)} \mathrm{H}
\end{gathered}
$$


The results show that the equivalent circuit model is able to accurately predict the input impedance of the antenna, which is relevant for designing a matching network.

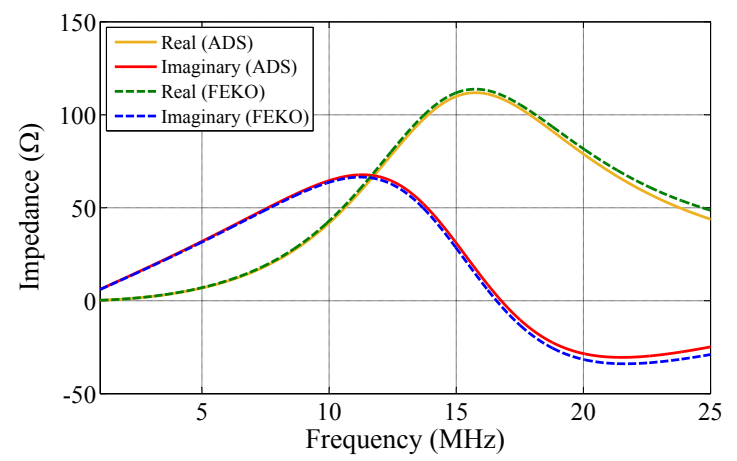

Fig. 7. Input impedance of the loop antenna, in freshwater, obtained in FEKO and ADS software.

TABLE I

VAlues of the Elements of the Equivalent CiRCuit

\begin{tabular}{|c|c|c|c|c|c|}
\hline$C_{0}^{\prime}$ & $R_{C 0}$ & $L_{0}$ & $C_{1}^{\prime}$ & $R_{C 1}$ & $L_{1}$ \\
\hline $20.57 \mathrm{pF}$ & $697.2 \Omega$ & $0.98 \mu \mathrm{H}$ & $69.54 \mathrm{pF}$ & $206.25 \Omega$ & $0.29 \mu \mathrm{H}$ \\
\hline
\end{tabular}

\section{RESUlTS}

This section presents the experimental results of the tests made in freshwater. These tests were performed in a freshwater tank located at INESC TEC, with $10 \mathrm{~m} \times 6 \mathrm{~m} \times 5.5 \mathrm{~m}$ $(L \times W \times H)$.

In order to compare the results with the simulations, two loop antennas (16 $\mathrm{cm}$ radius), with current baluns, were built (which are shown in Fig. 8). Current baluns were added to the antennas to reduce radiation on the coaxial cables. The antennas were placed at a depth of $2.5 \mathrm{~m}$ from the surface and on the centre of the pool. The distance was then measured between the antennas as they were separated. The conductivity of the water was $0.0487 \mathrm{~S} / \mathrm{m}$ at $25^{\circ} \mathrm{C}$.

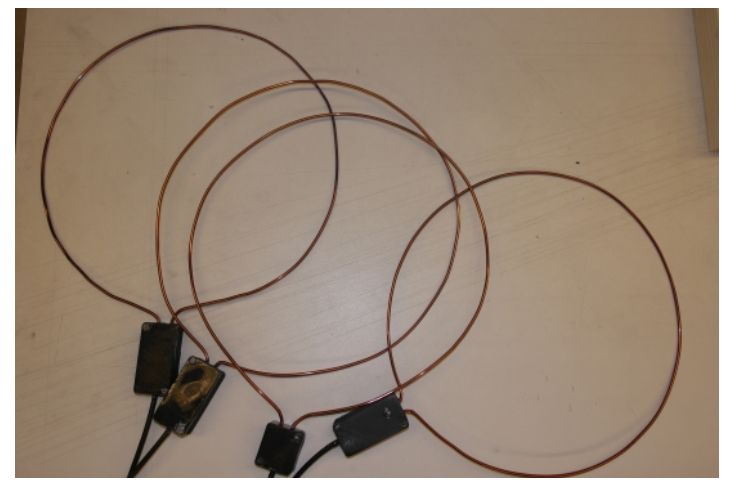

Fig. 8. Loop antennas.

\section{A. Power Parameters}

Fig. 9 shows a schematic of the setup used to perform the power parameter tests. The setup is based on a rope system, which allowed the distance between the antennas to be changed by pulling the ropes, and at the same time allowed the distance to be easily measured on one side of the pool. Fig. 10(a) and Fig. 10(b) show photos of the setup.

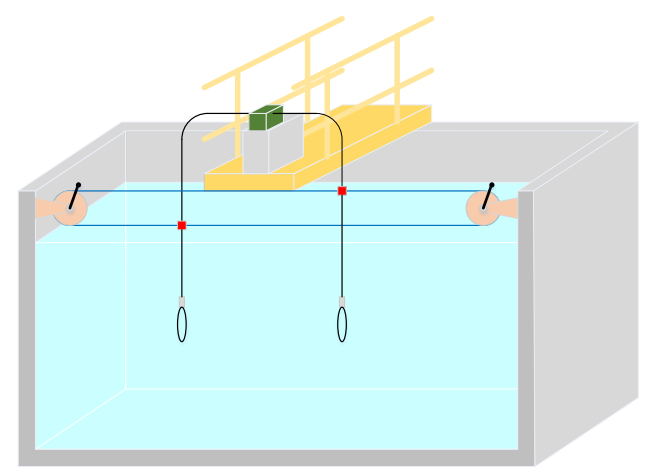

Fig. 9. Setup for the measurement of the power transmission parameter.

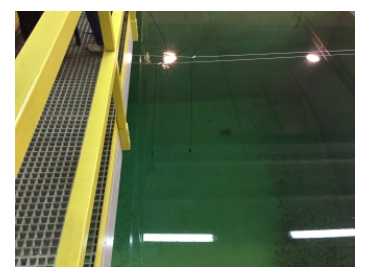

(a)

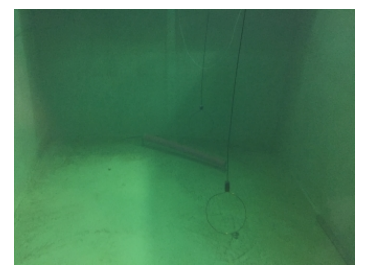

(b)
Fig. 10. Details of the setup used for the measurement of the power transmission parameter.

Fig. 11 shows the variation of the $S_{21}$ parameter, measured with a Vector Network Analyser (VNA) against the FEKO simulations, with the distance between the antennas. It can be observed that the $S_{21}$ parameter exhibited a good approximation to the simulation results. It was observed that the resonance frequency was $39 \mathrm{MHz}$, which is very close to the value obtained by simulation (Fig. 4(a)).

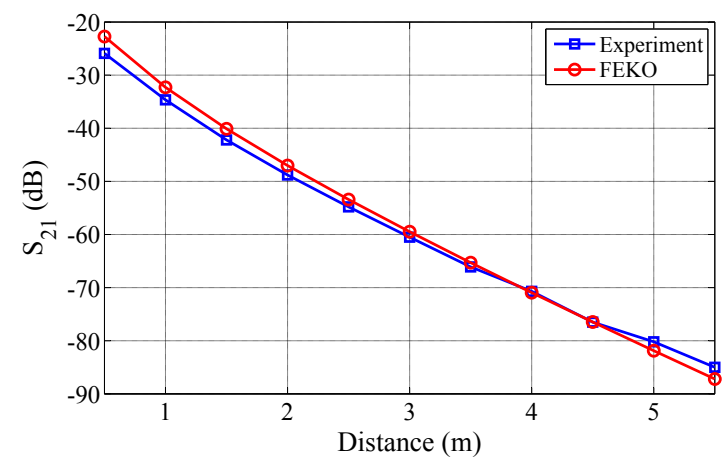

Fig. 11. $S_{21}$ as a function of the distance between the loop antennas. 


\section{B. Radiation Pattern}

The schematic of the setup for the measurement of the radiation patterns is shown in Fig. 12. One of the antennas was fixed to a wood structure and the antenna under test was allowed to rotate with the help of a protractor (as shown in Fig. 13(a) and Fig. 13(b)). Both antennas were connected to the VNA for the measurement of the $S_{21}$ parameter.

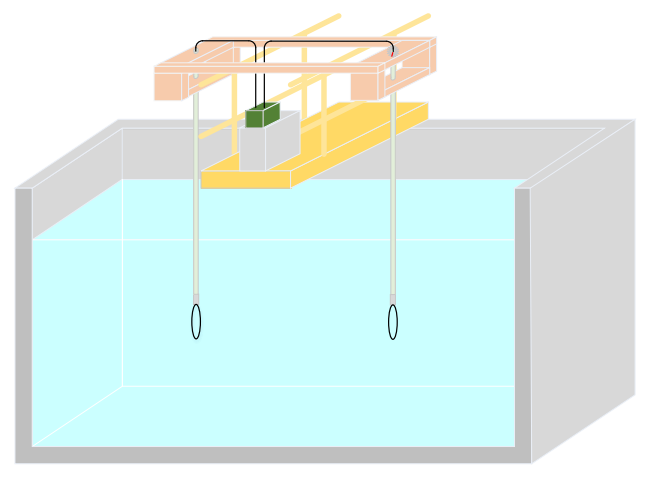

Fig. 12. Setup for the measurement of the radiation patterns.

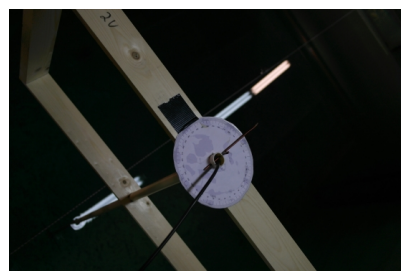

(a)

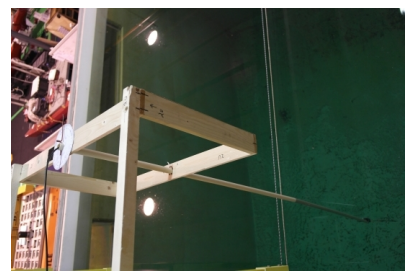

(b)
Fig. 13. Details of the setup used for the measurement of the radiation patterns.

The radiation pattern of the loop antenna was obtained in freshwater and the results, for the E-plane and H-plane, are presented in Fig. 14. It is seen that there is a good agreement between the measured and simulation results, both in the E-plane and on the H-plane, in terms of maximums and minimums of the radiation pattern.

\section{Conclusions}

In this work the characteristics of the loop antenna as a function of the conductivity of the medium were investigated. The main application addressed in this work was the use of radio communications in underwater systems.

An important result of this work is the drastic change in the orientation of the radiation pattern of the antennas between freshwater and seawater, which was verified through simulation. This indicates the need to take into account the properties of the medium and in particular its conductivity, at the design stage, as the direction of maximum radiation of an antenna will vary with the properties of the medium.

The experimental results in freshwater agree well with those obtained through FEKO simulations, both in terms of the

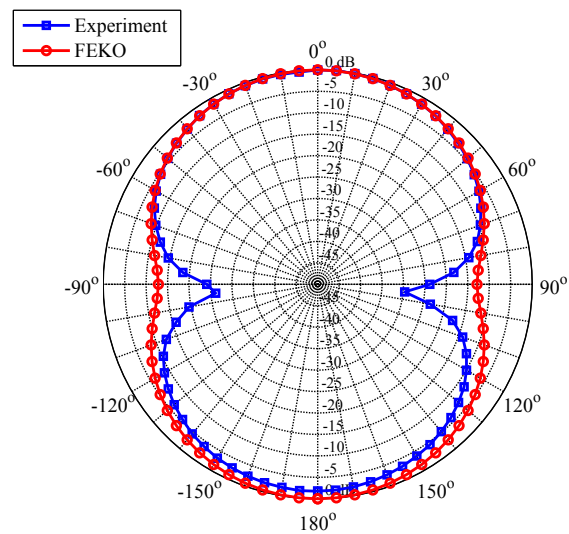

(a)

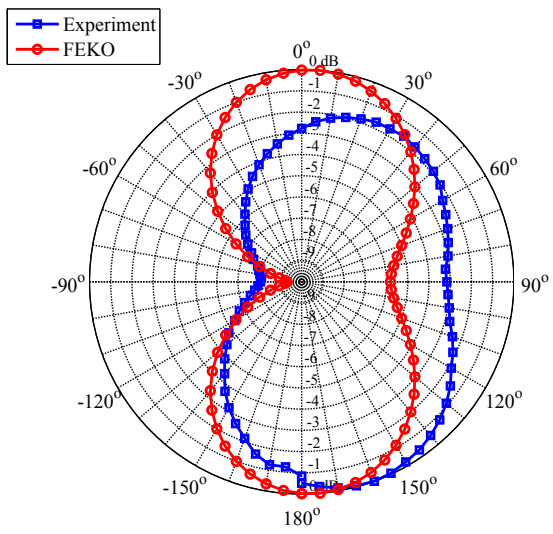

(b)

Fig. 14. Radiation patterns of the loop antenna: (a) E-plane and (b) H-plane.

transmission power parameter and radiation patterns. So we can conclude that it is possible to predict the operation of the antennas and improve their design with FEKO software.

As future work, we intend to repeat the same tests in seawater to evaluate the radiation pattern of the loop antenna in that environment and compare it with the FEKO simulations.

\section{ACKNOWLEDGMENT}

This work has been developed as part of project ENDURE - Enabling Long-Term Deployments of Underwater Robotic Platforms in Remote Oceanic Locations. ENDURE (PT02_Aviso4_0015) benefits from a $€ 218157$ grant from Iceland, Liechtenstein and Norway through the EEA Grants. The aim of the programme PT02 is to achieve a good environmental status, in accordance with the Marine Strategy Framework Directive and one of the expected outputs consists in the strengthening of the capacity of mobile remote monitoring ocean through the introduction of innovative supporting platforms. 


\section{REFERENCES}

[1] B. Benson, Y. Li, B. Faunce, K. Domond, D. Kimball, C. Schurgers, and R. Kastner, "Design of a low-cost underwater acoustic modem," Embedded Systems Letters, IEEE, vol. 2, no. 3, pp. 58-61, 2010.

[2] X. Che, I. Wells, G. Dickers, and P. Kear, "TDMA frame design for a prototype underwater RF communication network," Ad Hoc Networks, vol. 10, no. 3, pp. 317-327, 2012.

[3] M. Rhodes, "Underwater electromagnetic propagation: re-evaluating wireless capabilities," Hydro International, vol. 10 , no. 10, pp. 28-31, 2006.

[4] M. Rhodes et al., "Electromagnetic propagation in sea water and its value," in in Military Systems. SEAS DTC Technical Conference, Citeseer, 2007.

[5] F. Teixeira, P. Freitas, L. Pessoa, R. Campos, and M. Ricardo, "Evaluation of IEEE 802.11 Underwater Networks Operating at $700 \mathrm{MHz}$, $2.4 \mathrm{GHz}$ and $5 \mathrm{GHz}$," in Proceedings of the 9th ACM International Conference on Underwater Networks \& Systems, WUWNet'14, 2014.

[6] F. Teixeira, J. Santos, L. Pessoa, M. Pereira, R. Campos, and M. Ricardo, "Evaluation of Underwater IEEE 802.11 Networks at VHF and UHF Frequency Bands using Software Defined Radios," in Proceedings of the International Conference on Underwater Networks \& Systems, WUWNET '15, 2015.

[7] F. T. Ulaby and Michielssen, Fundamentals of Applied Electromagnetics. Pearson/Prentice Hall, 5 ed., 2013.

[8] E. A. Karagianni, "Electromagnetic Waves Under Sea: Bow-Tie Antennas Design for Wi-Fi Underwater Communications," Progress In Electromagnetics Research M, vol. 41, pp. 189-198, 2015.

[9] L. B. VK5BR, "Underwater Radio Communication," Originally published in Amateur Radio, 1987.

[10] S. Jiang and S. Georgakopoulos, "Electromagnetic wave propagation into fresh water," Journal of Electromagnetic Analysis and Applications, vol. 3, no. 07, p. 261, 2011.

[11] R. Somaraju and J. Trumpf, "Frequency, temperature and salinity variation of the permittivity of seawater," IEEE Transactions on Antennas and Propagation, vol. 54, no. 11, pp. 3441-3448, 2006.

[12] K. S. Cole and R. H. Cole, "Dispersion and absorption in dielectrics I. Alternating current characteristics," The Journal of Chemical Physics, vol. 9, no. 4, pp. 341-351, 1941.

[13] F. Franks, The physics and physical chemistry of water, vol. 1. Springer Science \& Business Media, 2012.

[14] D. K. Cheng, Field and wave electromagnetics, vol. 2. Addison-wesley New York, 1989.

[15] S. B. Wang, A. M. Niknejad, and R. W. Brodersen, "Circuit modeling methodology for UWB omnidirectional small antennas," IEEE Journal on Selected Areas in Communications, vol. 24, no. 4, pp. 871-877, 2006.

[16] Y. Liao, T. H. Hubing, and D. Su, "Equivalent circuit for dipole antennas in a lossy medium," IEEE Transactions on Antennas and Propagation, vol. 60, no. 8, pp. 3950-3953, 2012. 